\title{
Calidad del apego percibido hacia la figura PATERna, MATERNA Y PARES EN ADOLESCENTES INFRACTORES
}

\section{Quality of Perceived Attachment to the Paternal, Maternal, and Partner Figure in Adolescent Offenders}

\author{
Sergio Andrés Acosta Tobón ${ }^{1}$, Samuel Colorado Muñoz ${ }^{2}$, Sara Tahia \\ Eusse Gallego ${ }^{3}$, Jorge Mauricio Cuartas Arias ${ }^{4}$
}

doi: https://doi.org/10.17533/udea.rp.e346173

\section{Resumen}

El desarrollo evolutivo en la niñez y adolescencia está afectado por la percepción de la calidad del apego que el individuo atribuye a figuras paternales: madre - padre - pares. El objetivo central de este estudio fue estimar la calidad del apego percibido a figuras parentales y pares en adolescentes infractores privados de la libertad. El diseño metodológico fue cuantitativo de tipo observacional, transversal, retrospectivo y descriptivo, e implementó dos instrumentos: el autoinforme ad hoc (edad, escolaridad, delito, reincidencia, otros) y el Inventario de Apego con Padres y Pares validado para población adolescente en Colombia por Prado et al. (2006), que analiza tres dimensiones: comunicación, confianza y alienación. La muestra constó de 98 adoles-

Recibido: 2020-10-19/ Aceptado: 2020-12-28

Para citar este artículo en APA: Acosta, S., Colorado, S., Eusse, S. y Cuartas, J. (2020). Calidad del apego percibido hacia la figura paterna, materna y pares en adolescentes infractores. Revista de Psicología Universidad de Antioquia, 12(2), e346173. doi: https://doi.org/10.17533/udea.rp.e346173 centes hombres entre 14 y 19 años, privados de libertad por conductas punibles dolosas vinculados al Centro de Internamiento Preventivo "La Acogida" Antioquia (Colombia). La estadística descriptiva mediante medidas de tendencia central y análisis correlaciónales permitió identificar afectación negativa significativa en la percepción del apego hacia la figura paterna, en contraposición a la figura materna. La afectación en las dimensiones comunicación y confianza es más evidente en la delincuencia juvenil.

Palabras clave: afectividad; desarrollo afectivo; relaciones interpersonales; adolescencia; delincuencia juvenil.

1 Facultad de Educación y Ciencias Sociales, Programa Psicología, Tecnológico de Antioquia. Correo: sergio.acosta@tdea.edu.co; https://orcid.org/0000-0002-7042-7435.

2 Facultad de Ciencias Sociales, Programa Psicología, Institución Universitaria de Envigado; https://orcid.org/0000-0002-8383-4208.

3 Facultad de Ciencias Sociales, Programa Psicología, Institución Universitaria de Envigado; https://orcid.org/0000-0003-0808-9209.

4 Departamento de Psicología, Escuela de $\mathrm{Hu}$ manidades, Universidad EAFIT; https://orcid. org/0000-0001-9007-713X. 


\begin{abstract}
The evolutionary development in childhood and adolescence is affected by the perception of the quality of attachment that the individual attributes to parental figures: mother - father - peers. The central objective was to estimate the quality of the perceived attachment to parental figure in adolescent offenders deprived of liberty. The quantitative methodological design of an observational, transversal, retrospective and descriptive type implemented two instruments: a sociodemographic survey (age, schooling, crime, recidivism, others) and an Inventory of Attachment with Parents and Peers validated for the adolescent population in Colombia for Pardo and colleagues (2006), and reviewed three dimensions: communication, trust and alienation. The sample consists of 98 male adolescents
\end{abstract}

between 14 and 19 years old, deprived of liberty for intentional punishable conducts linked to the Preventive Internment Center "La Acogida" Antioquia (Colombia). The descriptive statistics through measures of central tendency and correlative analysis allowed to identify significant negative affectation in the perception of the attachment to the paternal figure, in contrast to the maternal figure. It should be noted that the effect on the communication and trust dimensions of the relationship is more pronounced in the case of juvenile delinquency.

Keywords: affectivity; affective development; interpersonal relationships; adolescence; juvenile delinquency

\section{Introducción}

El estudio del apego ha favorecido la comprensión de la naturaleza de las relaciones humanas e interpersonales (Policchi et al., 2013), desde el desarrollo evolutivo (Gago, 2014), especialmente en la niñez y juventud (Dávila, 2015). Según Bowlby, el apego es todo aquel comportamiento que beneficia proximidad y acercamiento entre personas (Garrido, 2006). Es decir, estudiar el apego permite concebir cómo una persona establece vínculos con otros. La teoría del apego revela la inclinación biológica ante el desarrollo de conductas y actitudes que impulsan la creación de vínculos afectivos y de proximidad, que permite generar sentimientos de seguridad y actitudes de protección (Camps, Carrillo y Cifre, 2014). El apego, por tanto, muestra la necesidad de establecer vínculos, especialmente seguros (Castillero, 2018). Según Delgado (2004), existen tres tipos de apego descritos por la psicóloga Mary Ainsworth:

Apego seguro: se construyen actitudes de confianza y seguridad para explorar el mundo.

Apego inseguro-evitativo: se muestran actitudes de independencia a la hora de explorar el mundo, acompañados de gestos de rechazo, generados por si- 
tuaciones dolorosas, dificultades emocionales y abandono propiciadas por el cuidador.

Apego inseguro ambivalente: se muestra preocupación ante la ausencia de la madre al momento de explorar el mundo, y muestra actitudes de indiferencia, generado por los cuidados inconsistentes y ambivalentes (Delgado, 2004).

La teoría del apego ha permitido a la psicología comprender a nivel patológico el vínculo afectivo e incidencia de este durante el desarrollo del ser humano (Beltrame, 2011). Así mismo, los estudios sobre apego han aportado a la psicología un amplio marco conceptual sobre el entendimiento de la regulación emocional (Mikulincer, Shave y Pereg, 2003) y como esta sirve al sujeto para adaptarse en sus relaciones durante su desarrollo (Guzmán et al., 2016).

Ahora bien, entendiendo la teoría del apego como un insumo para comprender cómo el ser humano instala relaciones con el mundo, la presente investigación tiene como objetivo identificar la calidad del apego percibido hacia la figura paterna, materna y los pares en adolescentes con conducta delictiva. Según Moneta (2014), por fuera de un apego seguro, el vínculo creado entre el padre, la madre y el hijo durante edades tempranas podrían constituirse en factor de riesgo para el desarrollo de trastornos mentales. Así pues, el presente estudio se propuso responder a la pregunta ¿el establecimiento de un tipo de apego percibido hacia el padre, la madre y los pares constituyen un factor asociado a la realización de conductas delictivas?

En ese orden de ideas, es necesario estudiar el apego como epicentro de análisis para identificar la génesis de la conducta antisocial o delincuencial de adolescentes en conflicto con la ley (Sarmiento et al., 2009). Según Camps, Carrillo y Cifre (2014), estudiar las relaciones entre el adolescente y cuidador, especialmente cuando se presenta abandono, indiferencia y necesidad de cuidado favorece el entendimiento sobre el impacto, tanto físico como psicológico, sobre la percepción y conductas divergentes o violentas. Es decir, a mayor apego seguro, mejores predictores psicológicos de la personalidad y patrones sociales en las relaciones intersubjetivas que harán parte de la vida durante la adolescencia y la adultez (González et al., 2010). Según Estrada (2009), dentro de las familias disfuncionales se crean vínculos irregulares entre el niño y sus padres en los cuales se desarrollan particularidades negativas en las con- 
ductas de los niños, aquellas que tienen efectos desadaptativos en el desarrollo social y mental durante la niñez y la adolescencia (Lecannelier, Ascanio, Flores y Hoffmann, 2011).

De acuerdo con lo anterior, entiéndase a la adolescencia como la fase del ciclo vital comprendida entre los diez y diecinueve años (Borras, 2013). Existen definiciones de adolescencia que delimitan su significado a entenderlo como la etapa de transición de la niñez a la adultez marcado por cambios biológicos (Lozano, 2014).

Ahora bien, la percepción de apego, durante la adolescencia, también sufrirá cambios (Horno, 2014). Esta transición desplaza la figura del cuidador hacia pares, quienes se convierten en el centro de las relaciones interpersonales (Crittenden, 2002). Respecto al papel del apego percibido en los actos delictivos, estudiar este fenómeno podría ayudar a entender cómo la influencia de los pares deriva en la sumisión hacia conductas delictivas (Vega, 2010).

En el Sistema de Responsabilidad Penal para Adolescentes colombiano, el artículo 139 registra que los adolescentes en conflicto con la ley son aquellas personas entre catorce y dieciocho años que realizan conductas punibles. Y, por conducta punible, se comprenden todas aquellas conductas delictivas o comportamientos que vayan en contra de un bien jurídico tutelado y que tienen, como consecuencia, una sanción (Niño y Rivero, 2019). Así pues, de manera específica, este estudio se interesó por los adolescentes en conflicto con la ley, partiendo de que el comportamiento delincuencial puede ser determinado por variables individuales psicológicas (teoría del apego), relacionadas directamente con los vínculos interpersonales que se establecen dentro de su entorno familiar y social (Sandoval y Martínez, 2008).

Por su parte, los indicadores de delincuencia juvenil arrojan estadísticas alarmantes respecto a la participación de adolescentes en conductas delincuenciales (Celedón et al., 2016). De acuerdo con la Oficina de Estadística y Justicia en Estado Unidos (2019), durante el 2018 fueron intervenidos y judicializados por los tribunales de menores un total de 744500 casos de delincuencia juvenil, en menores entre los 10 y 17 años. En España, el Instituto Nacional de Estadística (2019) indica que en el 2018 se procesaron judicialmente 13664 adolescentes entre 14 y 17 años. Según United Nations 
Children's Fund (2016), los indicadores de participación de adolescentes en delitos son significativamente altos. En Perú se registraron 10480000 casos, en Argentina 12076 000, en México 41942000 y en Brasil 58433000.

El Instituto Colombiano de Bienestar Familiar (2018) revela que entre el 2006 y el 30 de junio 2018, 251455 adolescentes han sido vinculados a procesos penales y solo en el 2018 se reportaron 9156 adolescentes privados de libertad en todo el territorio nacional. En Antioquia, entre el 2006 y el 30 de junio 2018 se reportaron 5045 adolescentes privados de la libertad por delitos, tales como: Tráfico, porte y fabricación de estupefacientes (41.88\%) y Hurto (20.42\%).

La alta incidencia de la adolescencia en las conductas delictivas deriva en la preocupación de la presente investigación acerca de cómo el apego se constituye en un factor criminológico (Feeney, 2011), dado que la Teoría del Apego estimula a pensar en los efectos de la relación primaria entre el niño y el cuidador (Dávila, 2015), y sobre cómo esa condición primaria puede influir en la calidad de las relaciones del desarrollo evolutivo (Bartol y Bartol, 2017) o predisponer hacia conductas delincuenciales (Neves, Faraco y Vieira, 2013). Según Cabrera y Gallardo (2013), las relaciones primarias generan consecuencias psicológicas que pueden desencadenar en un trastorno mental. En especial, se han descrito tendencias delincuentes en adolescentes cuando en la niñez se observa un tipo de apego inseguro ambivalente, basado en experiencias de abandono o abuso (González et al., 2010).

La presente investigación consideró los lineamientos éticos de investigación en Psicología contemplados en la ley 1090 de2006. Con el fin de garantizar el cumplimiento de los principios éticos se contó con el respaldo de la Facultad de Ciencias Sociales y con el programa de Psicología de la Institución Universitaria de Envigado, quienes asignaron evaluadores tanto del anteproyecto como del producto de la investigación. Así mismo, cabe resaltar que, en el desarrollo de la investigación, no se realizó una manipulación de variables que pudiera afectar la salud mental de los participantes. La investigación realizó una recopilación de datos e información y se expresaron los resultados de manera descriptiva, comunicando en todo momento los procesos que serían llevados a cabo y disponiendo por medio de un consentimiento informado 
una constancia de aceptación y participación (acorde a Resolución 8430 de 1993). A su vez, se contó con la autorización del Instituto Psicoeducativo de Colombia (IPSICOL), quien hace las veces de representante legal y autoriza la participación en la investigación, con pleno conocimiento de la naturaleza de los procedimientos y beneficios.

\section{Metodología}

El diseño fue cuantitativo (Curcio, 2002) y la variable central fue la percepción del apego hacia figuras parentales y pares, mediante un instrumento validado Colombia y con diseño estadístico ajustado que favoreciera alcanzar los objetivos de la investigación (Hernandez, 2014). A su vez, se identificaron las características socio-familiares de participantes mediante un autoinforme de elaboración conjunta entre los investigadores y los adolescentes (Mousalli, 2015).

Por otra parte, el diseño metodológico fue no-experimental, ya que incluye una recolección de datos en campo que no tuvo intervenciones en la muestra o manipulación de los datos recolectados por parte de los investigadores (Naupas, Valdivia, Palacios y Romero, 2018). En ese orden de ideas, el diseño metodológico tuvo las siguientes características: observacional, retrospectivo, descriptivo y transversal.

\section{Población y muestra}

El estudio tuvo una muestra de 98 adolescentes hombres con edades superiores a los 14 ańos que están privados de la libertad por la autoridad judicial del Sistema de Responsabilidad Penal para adolescentes y que son vinculados en el Centro de Internamiento Preventivo (CIP) La Acogida de la ciudad de Medellín y de otros municipios de Antioquia. La autoridad judicial en los términos del artículo 181 de la Ley 1098 de 2006, a través de un juez de control de garantías, les impone esta medida de privación de libertad a personas que, siendo mayores de 14 años y menores de 17 años y 11 meses, hayan sido capturadas en flagrancia o remitidos por denuncia penal como indiciados en una presunta conducta delictiva. 
Teniendo presente las dificultades de acceso a la población objeto de estudio, se realizó un muestreo no probabilístico por conveniencia (López, 2004). Esta técnica se caracteriza porque permite a los investigadores seleccionar la muestra según criterios establecidos internamente y las posibilidades de disponibilidad que se determinan a conveniencia del estudio (Hernandez, 2014).

\section{Técnicas e instrumentos}

Autoinforme ad hoc: se realizó una caracterización de la población donde se formuló una serie de variables que favorecieron los análisis, valorando algunos aspectos personales, familiares y del entorno que pudieran representar posibles factores predictores en la población evaluada. El autoinforme ad hoc permitió evaluar tres ámbitos: datos de identificación, información judicial e información familiar. En el primer ámbito, se verifican aspectos personales como la edad, el grado de escolaridad, el género y el estrato socioeconómico; en el segundo, se indagó sobre información de la situación judicial como lo es el tipo de delito, si hay reincidencia y si se encuentra sancionado o en proceso judicial; $y$, por último, se examinaron aspectos familiares relevantes, como el lugar de procedencia, las personas con las que vive el encuestado y la composición familiar. Esta información contribuyó a la clasificación y tipología de la población de estudio para el análisis de correlaciones posteriores.

Inventario de apego para padres y pares: el Inventario de Apego con Padres y Pares para Adolescentes (IPPA) es un instrumento creado por Armsden y Greenberg (1987) para medir apego percibido en adolescentes mediante la inclusión de tres dimensiones: confianza, comunicación y alienación (Delgado et al., 2016). Entiéndase: la confianza como la confidencia y respeto mutuo que el adolescente tiene hacía las figuras paternales; la comunicación como el nivel interacción verbal y la calidad percibida hacia las figuras paternas; y, la alienación como el sentimiento de aislamiento y alienación percibida hacia las figuras parentales.

Cada una de las dimensiones se miden mediante 75 ítems formulados en escala Likert, en la que 1 corresponde a "nunca" y 5 a "siempre o casi siempre". El instrumento cuenta con un nivel de confiabilidad o Alfa de Cronbach en 
un rango entre 0.72 y 0.92 (Delgado et al., 2016). El IPPA fue validado en Colombia por Prado, Pineda, Carrillo y Castro (2006), mediante un estudio que se realizó en la Universidad de los Andes (Bogotá) con 1435 adolescentes colombianos, con edades comprendidas entre los 15 a 20 años, de los cuales el $48.9 \%$ eran masculinos y el $51.1 \%$ femeninos. Como datos relevantes, los participantes pertenecían a distintos niveles socio económico: bajo $(14 \%$,$) ,$ medio (64\%) y alto (el 22\%). El Ippa posee un índice de consistencia interna para la escala de la madre de 0.90 , para la del padre de 0.93 y para la de los pares de 0.90. El índice de adecuación muestral Kaiser Meyer Olkin revela la validez del IPPA mediante análisis factorial confirmatoria de componentes principales con rotación de tipo Varimax.

\section{Resultados y discusión}

\section{Caracterización sociodemográfica de los adolescentes del CIP La Acogida}

Los resultados del autoinforme ad hoc han permitido caracterizar la población participante, reflejando datos relevantes para la presente investigación. Los resultados indican que, entre los adolescentes Privados de la Libertad (PL), el 45.9\% tienen 17 ańos, seguido de un $24.5 \%$ que tienen 16 ańos, sugiriéndose que la adolescencia media (16-17 ańos), tal como lo indica la prevalencia de trastornos de la conducta, es un momento relevante en el ciclo vital para incurrir en conductas delictivas (Garrido, León, López y Ojeda, 2020) (ver Tabla 1). Entre los resultados se encuentran factores como: un nivel socioeconómico bajo, un gran número de familias monoparentales y una posible presencia de problemas psicopatológicos en los padres, lo cual aumenta la probabilidad de que se manifiesten trastornos conductuales que desemboquen en conductas criminales (Bragado, Bersabé y Carrasco, 1999). 
Calidad del apego percibido hacia la figura paterna, materna y pares en adolescentes infractores

\section{Tabla 1.}

Distribución de los datos de acuerdo con la variable Edad

\begin{tabular}{ccc}
\hline Edad & Frecuencia & $\%$ \\
\hline-15 años & 10 & 10.2 \\
16 años & 24 & 24.5 \\
17 años & 45 & 45.9 \\
+ de 18 años & 19 & 19.4 \\
Total & 98 & 100 \\
\hline
\end{tabular}

Con respecto al estrato socioeconómico, cabe resaltar que no se obtuvieron resultados para los estratos 5 y 6 , por lo que no se pueden hacer inferencias al respecto. Sin embargo, el $53.1 \%$ manifiestan pertenecer a estrato 2 y el $23 \%$ al estrato 1 (ver Tabla 2). Es decir, en estudio sobre delincuencia juvenil se confirma la hipótesis de la condición socioeconómica como factor de vulnerabilidad a la delincuencia (Jimenez y Rene, 2005). Paramo (2011), en un estudio sobre delincuencia juvenil, indica que el estrato socio económico bajo puede ser un factor de riesgo a la delincuencia debido a la relación entre la condición y la vulneración de los derechos desde tempranas edades.

Tabla 2.

Distribución de los datos de acuerdo con el estrato socioeconómico

\begin{tabular}{ccc}
\hline Estrato & Frecuencia & $\%$ \\
\hline Estrato 1 & 23 & 23.5 \\
Estrato 2 & 52 & 53.1 \\
Estrato 3 & 18 & 18.4 \\
Estrato 4 & 5 & 5.1 \\
Total & 98 & 100 \\
\hline
\end{tabular}

Al revisar la tipología de delito, los datos indican una alta frecuencia en Hurto agravado y calificado (36.7\%), seguido de Homicidio (22,4\%) (ver Tabla 3). Estos datos dialogan con los indicadores de delincuencia juvenil del Instituto Colombiano de Bienestar Familiar (ICBF) (2018), en particular para Antioquia, donde se ha determinado entre el 2006 y el 2018 un total de 5045 
adolescentes PL por Hurto. Es decir, el 20.42\% de la totalidad de los adolescentes PL ingresa al SRPA por este delito, mostrando una alta tasa de participación en esta tipología criminal. Por otra parte, al revisar los datos de reincidencia, el $33.7 \%$ expresó reincidencia en conductas delictivas, indiferente al motivo de ingreso al SRPA. Según Molina y Graciela (2018), la reincidencia en la delincuencia juvenil obedece a la vinculación a grupos sociales que, debido al patrón relacional, se constituyen en agentes activos que condicionan los patrones comportamentales y criminológicos; los pares no solo constituyen figuras donde se establecen lazos afectivos, sino que son actores modeladores de la conducta; por lo tanto, cuando un adolescente retorna a su núcleo social perpetúa las conductas aprendidas y reincide en el delito, especialmente en el Hurto.

\section{Tabla 3.}

Distribución de los datos de acuerdo con el tipo de delito

\begin{tabular}{ccc}
\hline Delito & Frecuencia & $\%$ \\
\hline Secuestro Extorsión & 8 & 8.2 \\
Porte Ilegal de armas & 13 & 13.3 \\
Hurto agravado y calificado & 36 & 36.7 \\
Trafico de SPA & 7 & 7.1 \\
Homicidio & 22 & 22.4 \\
Violencia intrafamiliar & 12 & 12.2 \\
Total & 98 & 100 \\
\hline
\end{tabular}

Por otra parte, la muestra revela una tipología familiar donde el $27.6 \%$ hace parte de una familia nuclear, seguido por el $25.5 \%$ que pertenece a una familia monoparental materna lo que indica ausencia de una figura paternas en las familias monoparentales. Las relaciones parentales son fundamentales para que el adolescente tenga un desarrollo adecuado; sin embargo, la inexistencia de la relación afectiva genera conflicto y aumenta la probabilidad de delincuencia (Miron, Luengo, Sobral y Otero, 1988). Es decir, mientras el 2\% manifiesta no tener relación con su figura materna, el 30.6\% de la totalidad de los participantes no cuenta con vínculo paterno, mostrando un alto índice de abandono por parte de la figura paterna (Tabla 4). 
Calidad del apego percibido hacia la figura paterna, materna y pares en adolescentes infractores

\section{Tabla 4.}

Distribución de los datos de acuerdo con variable de tipología familiar

\begin{tabular}{cc}
\hline Tipología familiar & Distribución \\
\hline Nuclear & $27.55 \%$ \\
Extensa & $15.31 \%$ \\
Extendida & $14.29 \%$ \\
Monoparental materna & $25.51 \%$ \\
Otros & $17.35 \%$ \\
\hline
\end{tabular}

La teoría del apego indica que cuando el vínculo se logra de manera segura, el adolescente desarrolla habilidades, sentimientos, deseos, creencias, expectativas prosociales y reconoce derechos propios y de los demás. Sin embargo, cuando desde edades tempranas se experimentan experiencias de abandono se reducen las posibilidades de desarrollar estas habilidades, y se convierte en un factor de riesgo a conductas antisociales y delictivas (Rendon y Rodriguez, 2016). En síntesis, los efectos de relaciones disfuncionales y, en este caso, de abandono de la figura paterna, pueden constituirse en aspectos relevantes en el desarrollo del juicio moral y, por lo tanto, en factores de riesgo a considerar en los estudios acerca de los adolescentes con problemas de conducta y con conductas delincuenciales (Nieri, 2017).

En resumen, podemos indicar que las características sociodemográficas de la población participante revelan adolescentes en su mayoría entre 16 y 17 años, provenientes de estratos socioeconómicos 1 y 2, con una alta frecuencia en delitos como Hurto agravado - calificado y Homicidio, con altos índices de reincidencia. Respecto a la tipología familiar, predomina la familia Nuclear y Monoparental Materna, evidenciando el abandono paterno en un porcentaje significativo.

\section{Percepción del apego hacia padres y madres}

Los resultados de las puntuaciones directas muestran que para los jóvenes el coeficiente de confianza y comunicación con la madre son altos, mientras que el coeficiente de asimetría de alienación es bajo, lo cual sugiere que el vínculo 
de apego con la madre es frecuentemente percibido como principal, mientras que el vínculo con la figura paterna y los pares es percibido como figuras de apego secundarias (Dávila, 2015).

Además, se resalta el hecho de que los resultados de las puntuaciones directas concernientes a la figura paterna y los pares, es más bajo que el de la madre, sin embargo, guardan similitud en sus puntuaciones, lo que significa que, para los jóvenes, las dimensiones de confianza, comunicación y alienación se presentan de la misma forma percibiendo el apego con los padres muy similar a como lo perciben con los pares. Siguiendo con lo anterior, podemos decir que en el vínculo entre el adolescente y la madre es mejor percibida la relación de apego, sin embargo, el vínculo en la relación con el padre y con los pares es más influyente en las conductas delincuenciales, a pesar de que hay una mayor presencia de la madre (ver Tabla 5).

\section{Tabla 5.}

Percepción de apego hacia la figura materna, paterna y pares de acuerdo a resultados IPPA

\begin{tabular}{ccccccc}
\hline Percepción Madres & $\mathrm{N}$ & Mínimo & Máximo & Suma & Media & Desv. típ. \\
\hline Percepción Confianza & 98 & 1 & 5 & 421 & 4.3 & 0.5 \\
Percepción Comunicación & 98 & 3 & 5 & 395 & 4.0 & 0.4 \\
Percepción Alienación & 98 & 1 & 5 & 250 & 2.6 & 0.7 \\
Global & 98 & 2 & 4 & 356 & 3.4 & 0.3 \\
\hline Percepción Padres & $\mathrm{N}$ & Mínimo & Máximo & Suma & Media & Desv. típ. \\
\hline Percepción Confianza & 98 & 0 & 4 & 290 & 3.0 & 1.1 \\
Percepción Comunicación & 98 & 0 & 5 & 339 & 3.5 & 1.2 \\
Percepción Alienación & 98 & 0 & 5 & 228 & 2.3 & 1.0 \\
Global & 98 & 0 & 4 & 285 & 2.9 & 1.0 \\
\hline Percepción Pares & $\mathrm{N}$ & Mínimo & Máximo & Suma & Media & Desv. típ. \\
\hline Percepción Confianza & 98 & 0 & 5 & 351 & 3,6 & 1,0 \\
Percepción Comunicación & 98 & 0 & 5 & 339 & 3,5 & 1,1 \\
Percepción Alienación & 98 & 0 & 4 & 263 & 2,7 & 0,7 \\
Global & 98 & 0 & 5 & 317 & 3,2 & 0,7 \\
\hline
\end{tabular}


Incidencia del vinculo paterno filial en la estructuración del afecto de adolescentes del SRPA

Dentro de los resultados obtenidos por medio del coeficiente de correlación de Pearson, hay dos resultados que llaman la atención: el primero es la correlación directamente proporcional que se encuentra entre la percepción de confianza y comunicación hacia la figura paterna, que es de 0.95 (ver tabla 6), el cual resalta la importancia y la influencia del vínculo paterno para los adolescentes; este vínculo afectivo se configura en la relación padre y niño, lo cual afecta el desarrollo sano y equilibrado del menor, por lo cual el vínculo parental afecta la calidad de vida del adolescente y se considera una función enriquecida de experiencias gratificantes para el adolescente (Perez y Arrazola, 2013). Según lo anterior, cabe resaltar que dentro del estudio sus resultados apuntan a que hay una mejor percepción en torno a la calidad del vínculo paterno en esa relación padre - hijo.

Si bien se da cuenta de una percepción positiva con respecto al vínculo maternal, esta investigación contribuye a las discusiones de la percepción de apego paterno en los sujetos del estudio. Al respecto, desde la infancia, los niños se apoyan del modelo masculino para su desarrollo, para mejorar procesos adaptativos entre pares de acuerdo con las exigencias del entorno, y para desarrollar patrones sobre la regulación y contingencia conductual en la norma social. Adicionalmente, una labor esencial de este vínculo es la de proveer seguridad frente a situaciones de peligro o en las que el niño o adolescente se siente inseguro. De otro lado, este vínculo afectivo paternal refuerza las habilidades sociales y emocionales en la infancia y adolescencia.

Estos resultados coinciden con lo encontrado por Delgado et al. (2016), quienes en su análisis correlacional presentado con el IPPA encontraron resultados directamente proporcionales entre las variables de confianza y comunicación. Asimismo et al. (2006), encontraron los mismos resultados con la presente investigación $(0,77)$. Se puede decir que el abandono por parte de la figura paterna trae consigo algunas consecuencias como baja autoestima, baja autoconfianza y frustración, por lo que se busca reemplazar ese vínculo ausente con alguna otra figura o cuidador que lo supla. Asimismo, se pueden 
desarrollar conductas rebeldes, debido a que no se sienten comprendidos y esto tiende a acentuarse durante la adolescencia, convirtiéndose en un factor de riesgo con respecto a la realización de conductas delincuenciales; además, se presenta una alta probabilidad de desarrollar algún trastorno psicológico. Por esto se debe considerar que, dentro de la caracterización de la población, es que se evidencia que el $70.4 \%$ de la muestra cuenta con un vínculo paterno activo, lo cual puede ser determinante con relación a los resultados obtenidos respecto a la percepción de confianza y comunicación con la figura paterna.

Ahora bien, se recomienda que en futuras investigaciones se indague si esa percepción positiva del vínculo afectivo frente a la comunicación y la confianza con el padre actúa como un factor protector frente a la incidencia en conductas delincuenciales o, por el contrario, si actúa como un factor de riesgo para estos adolescentes que se encuentran en el SRPA.

\section{Incidencia del vinculo relacional con los pares en jóvenes del SRPA}

Otro resultado que vale la pena resaltar es la correlación directamente proporcional que se encuentra entre la percepción de confianza y comunicación hacia el vínculo afectivo que desarrolla el adolescente con sus pares, el resultado encontrado es de 0.84 (ver Tabla 6). Este dato es importante debido a que la interacción con los pares es esencial para que se dé el desarrollo normal del adolescente, dado que este se encuentra en una etapa de desarrollo en la cual se desliga de sus padres y ese desprendimiento se realiza en áreas como las creencias e ideologías que sus padres le han enseñado desde su nacimiento — sean políticas, religiosas, sociales e incluso conductuales-. De este modo, comienza un proceso de autoconocimiento, en el cual el adolescente busca ser aceptado, incluido y reconocido por sus pares; y, por lo general, los grupos de adolescentes crean sus propias normas y reglas (Urrego et al., 2014). Todo esto se fundamenta en la confianza que se deposita en la persona que es igual (par) al adolescente; sin embargo, cuando el adolescente no cuenta con adultos de confianza que lo guíe y asesore en la toma de decisiones responsables, estos grupos de pares se pueden convertir en un factor decisivo a la hora de cometer conductas delincuenciales. 
Estos resultados confirman la importancia que tiene la relación afectiva de los pares con respecto al proceso de emancipación y maduración, tanto personal como social, especialmente si notamos que la percepción de este vínculo relacional es de importancia para los adolescentes pertenecientes al SRPA. A propósito, es importante tomar en cuenta que el vínculo afectivo parental y el de los pares no se contradicen el uno al otro, sino que, por el contrario, se completan y corroboran recíprocamente (Ayestarán, 1987).

\section{Tabla 6.}

Coeficientes de correlación de Pearson IPPA

\begin{tabular}{|c|c|c|c|c|c|c|c|c|c|c|c|}
\hline & \multicolumn{2}{|c|}{ Percepción } & \multicolumn{3}{|c|}{ Madre } & \multicolumn{3}{|c|}{ Padre } & \multicolumn{3}{|c|}{ Pares } \\
\hline & & & $\mathrm{C}$ & Co & A & $\mathrm{C}$ & Co & A & $\mathrm{C}$ & Co & A \\
\hline \multirow{6}{*}{ Madre } & \multirow{2}{*}{$\mathrm{C}$} & C. Pearson & 1 & $.517^{* *}$ & -.101 & $.199^{*}$ & .183 & .099 & .054 & .012 & .046 \\
\hline & & Sig. (bilateral) & & .000 & .320 & .049 & .071 & .331 & .599 & .910 & .656 \\
\hline & \multirow{2}{*}{ Co } & C. Pearson & $.517^{* *}$ & 1 & $-.317^{* *}$ & .131 & .167 & -.093 & .156 & .119 & -.184 \\
\hline & & Sig. (bilateral) & .000 & & .001 & .198 & .101 & .360 & .124 & .244 & .070 \\
\hline & \multirow{2}{*}{ A } & C. Pearson & -.101 & $-.317^{* *}$ & 1 & -.083 & -.069 & $.306^{*}$ & .041 & .128 & $.287^{* *}$ \\
\hline & & Sig. (bilateral) & .320 & .001 & & .414 & .499 & .002 & .688 & .209 & .004 \\
\hline \multirow{6}{*}{ Padre } & \multirow{2}{*}{$\mathrm{C}$} & C. Pearson & $.199^{*}$ & .131 & -.083 & 1 & $.950^{* *}$ & $.562^{* *}$ & .192 & $.203^{*}$ & .136 \\
\hline & & Sig. (bilateral) & .049 & .198 & .414 & & .000 & .000 & .058 & .045 & .182 \\
\hline & \multirow{2}{*}{ Co } & C. Pearson & .183 & .167 & -.069 & $.950^{* *}$ & 1 & $.572^{* *}$ & $.237^{*}$ & $.275^{* *}$ & .126 \\
\hline & & Sig. (bilateral) & .071 & .101 & .499 & .000 & & .000 & .019 & .006 & .216 \\
\hline & \multirow{2}{*}{ A } & C. Pearson & .099 & -.093 & $.306^{* *}$ & $.562^{* *}$ & $.572^{* *}$ & 1 & $.204^{*}$ & $.291^{* *}$ & $.211^{*}$ \\
\hline & & Sig. (bilateral) & .331 & .360 & .002 & .000 & .000 & & .044 & .004 & .037 \\
\hline \multirow{6}{*}{ Pares } & \multirow{2}{*}{$\mathrm{C}$} & C. Pearson & .054 & .156 & .041 & .192 & $.237^{*}$ & $.204^{*}$ & 1 & $.844^{* *}$ & -.047 \\
\hline & & Sig. (bilateral) & .599 & .124 & .688 & .058 & .019 & .044 & & .000 & .644 \\
\hline & \multirow{2}{*}{ Co } & C. Pearson & .012 & .119 & .128 & $.203^{*}$ & $.275^{* *}$ & $.291^{* *}$ & $.844^{* *}$ & 1 & .046 \\
\hline & & Sig. (bilateral) & .910 & .244 & .209 & .045 & .006 & .004 & .000 & & .656 \\
\hline & \multirow{2}{*}{ A } & C. Pearson & .046 & -.184 & $.287^{*}$ & .136 & .126 & $.211^{*}$ & -.047 & .046 & 1 \\
\hline & & Sig. (bilateral) & .656 & .070 & .004 & .182 & .216 & .037 & .644 & .656 & \\
\hline
\end{tabular}




\section{Discusión}

Por lo anterior, es relevante estudiar las formas de apego en el desarrollo de la personalidad, ya que constituyen un eje central para comprender el impacto que tiene el desarrollo de habilidades de autoregulación que se adquieren a través de los procesos de interacción de los niños con las figuras parentales y con los pares en las primeras etapas del desarrollo. A partir de los hallazgos obtenidos en esta investigación, se ha observado consistencia con diferentes estudios recientes en torno a los trastornos externalizantes en la adolescencia y el impacto de la teoría del apego para explicar el curso y la evolución de los trastornos de la personalidad y de la psicopatología (Ramos et al., 2016; Sharp y Fonagy, 2015). Al respecto, se ha documentado que formas de apego inseguras pueden predisponer el desarrollo del Trastorno Límite de la Personalidad (TLP), de un patrón inestable de relaciones interpersonales y, en consecuencia, de una gran inestabilidad afectiva, impulsividad y hostilidad. Al momento, el apego ansioso y ambivalente percibido ha resultado ser uno de los más frecuentemente asociados con el TLP (Clarkin y De Panfilis, 2013; Steele et al., 2015). Adicionalmente, el apego inseguro ha sido asociado con experiencias negativas en el aprendizaje de las primeras relaciones de apego, y delinea una capacidad limitada para confiar en los pares y en las figuras parentales, promoviendo el desarrollo de conductas violentas y psicopatología.

No obstante, aunque hasta ahora diferentes estudios han analizado de manera general cómo las alteraciones en el apego percibido contribuyen a desarrollar trastornos de la personalidad (Fossati et al., 2015; Levy et al., 2015), aún no hay consenso en cuanto a si los estilos de apego explican de manera directa trastornos de personalidad específicos. Como se mencionó, algunos investigadores han sugerido que el apego inseguro ambivalente está más estrechamente asociado con el TlP (Barone, Fossati y Guiducci, 2011; Beeney et al., 2015), mientras que el apego evitativo ha estado más ligado con los trastornos de personalidad paranoides, esquizotípicos y antisociales (Bakermans-Kranenburg y van IJzendoorn, 2009; Levy et al., 2015; Garofalo y Bogaerts, 2019).

Con respecto a lo anterior, se requiere estudiar en futuras investigaciones cómo se complementan el vínculo afectivo parental con respecto al vínculo 
afectivo con los pares, cuál de las dos tiene mayor fuerza a la hora de la toma de decisiones con respecto a la incidencia de conductas delincuenciales.

\section{Conclusiones}

La caracterización sociodemográfica indica que los adolescentes participantes tienen edades entre 14 y 19 años, de los cuales el 76.5\% son de estratos 1 y 2. Se observa, además, la participación en conductas delincuenciales como secuestro, extorsión, porte ilegal de armas, hurto agravado y calificado, tráfico de SPA, homicidio, violencia intrafamiliar. Asimismo, los índices de reincidencia son de un $33.5 \%$. Dentro de la tipología familiar predomina la familia nuclear, seguida de la monoparental materna.

Frente al vínculo materno y paterno se concluye que el $98.2 \%$ tiene un vínculo activo con la madre y el $69.4 \%$ lo tienen con una figura paterna. Según lo anterior, los resultados de las puntuaciones directas obtenidas del IPPA exponen que los adolescentes tienen mayor confianza y comunicación con la madre, mientras que con el padre y los pares es baja, esto significa que, si bien entre los adolescentes el vínculo con la madre indica mayor percepción del apego, la confianza y la comunicación que tengan con el padre y los pares es la que tiene mayor incidencia en la realización de conductas delincuenciales.

Dentro de estas relaciones de apego, las dimensiones de confianza y comunicación son directamente proporcionales, demostrando que, a mayor confianza mayor comunicación dentro del vínculo con el padre y con los pares. Siguiendo lo anterior, podemos concluir que es notable la importancia que tiene sostener relaciones basadas en la confianza y la comunicación con los padres y con los pares, ya que ejercen un papel crucial en el desarrollo normal del adolescente.

Esta investigación permitió dimensionar la calidad del apego percibido entre los adolescentes y sus figuras de apego y pares, posibilitando conocer la importancia que estas figuras de apego tienen en el desarrollo evolutivo del adolescente, y la incidencia que asumen en la creación de conductas antisociales y la comisión de un delito. El estudio de la teoría del apego representa un aporte relevante para el acercamiento a una problemática psicosocial, como 
lo son los adolescentes infractores. Su abordaje permite el esclarecimiento de procesos de resocialización y tratamiento de esta población, que se ha visto como una problemática en crecimiento a nivel mundial.

\section{Referencias}

Ayestarán, S. (1987). El grupo de pares y el desarrollo psicosocial del adolescente. Estudi general: Revista de la Facultat de Lletres de la Universitat de Girona, (7), 123-136.

Bakermans-Kranenburg M. y van IJzendoorn M. (2009). The First 10,000 Adult Attachment Interviews: Distributions of Adult Attachment Representations in Clinical and Non-Clinical Groups. Attachment \& Human Development, 11(3), 223-263. doi: 10.1080/14616730902814762.

Barone L., Fossati A. y Guiducci V. (2011). Attachment Mental States and Inferred Pathways of Development in Borderline Personality Disorder: A Study Using the Adult Attachment Interview. Attachment \& Human Development, 13(5), 451-469. doi: 10.1080/14616734.2011.602245.

Bartol, C. y Bartol, A. (2017). Comportamiento criminal, una perspectiva psicologica. Cuidad de Mexico : Person educacion de Mexico S.A.

Beeney J., Stepp S., Hallquist M., Scott L., Wright A., Ellison W., Kimberly N. y Pilkonis P. A. (2015). Attachment and Social Cognition in Borderline Personality Disorder: Specificity in Relation to Antisocial and Avoidant Personality Disorders. Personality Disorders: Theory, Research, and Treatment, 6, 207-215. doi:10.1037/per0000110.

Beltrame, G. (2011). Bases Neurobiológicas del Apego. Ciencias Psicologicas, 5(1), 69-81.

Bragado, C., Bersabé, R. y Carrasco, I. (1999). Factores de riesgo para los trastornos conductuales, de ansiedad, depresivos y de eliminación en niños y adolescentes. Psicothema, 11(4), 939-956.

Cabrera, J. y Gallardo, R. (2013). Psicopatía y apego en los reclusos de una cárcel chilena. Anuario de Psicologia/The UB Journal of Psycholog, 43(1), 83-99.

Camps-Pons, S., Castillo, J. y Cifre, I. (2014). Apego y psicopatología en adolescentes y jóvenes que han sufrido maltrato: implicaciones clinicas. Clinica y Salud, 25(1), 67-74. 
Calidad del apego percibido hacia la figura paterna, materna y pares en adolescentes infractores

Clarkin, J. y de Panfilis, C. (2013). Developing Conceptualization of Borderline Personality Disorder. Journal of Nervous and Mental Disease, 201(2), 88-93.

Castillero, O. (2018). Psicologia y Mente. Obtenido de Psicologia y Mente: https:// psicologiaymente.com/biografias/john-bowlby

Celedon, J., Cogollo, M., Garcia, B., Miranda , M. y Martinez, P. (2016). Estilos de apego en un grupo de jóvenes con rasgos antisociales y psicopáticos. Encuentros, 14(1), 151-165.

Crittenden, P. (2002). Transformaciones en Relaciones de Apego en la Adolescencia. Revista de Psicoterapia, 12(48), 33-62.

Curcio, L. (2002). Investigacion cuantitativa una perspectiva epistemologica y metodologica. Armenia, Colombia: KINESIS.

Dávila, Y. (2015). La Influencia de la Familia en el Desarrollo de Apego. Revista de la Universidad de Cuenca, 57, 121-130. ISSN 1390-9657.

Delgado, L., Penelo, E., Fornieles, A., Gasca, C. y Ollé, M. (2016). Estructura factorial y consistencia interna de la versión española del Inventario de Apego a Padres y Pares para Adolescentes (IPPA). Universitas Psychologica, 15(1), 327-338.

Delgado, O. (2004). Estado actual de la teoría del apego. Revista de Psiquiatría y Psicología del Niño y del Adolescente, 4(1), 65-81.

Estrada, P. (2009). Investigacion sobre el estilo de apego en nińos prescolares pertenecientes a familias multiproblematicas. Subjetividad y Procesos Cognitivos, 13(1), 45-54.

Feeney, J. (2011). Adult Attachment and Conflict Behavior: Delineating the Links. Acta de Investigación Psicológica - Psychological Research Records, 1(2), 233-254.

Fossati A., Krueger R., Markon K., Borroni S., Maffei C. y Somma A. (2015). The DSM-5 Alternative Model of Personality Disorders From the Perspective of Adult Attachment: A Study in Community-Dwelling Adults. Journal of Nervous and Mental Disease 203(4), 252-258. doi:10.1097/NMD.0000000000000274.

Gago, J. (2014). Teoria del apego. El Vinculo. Bilbao: Agintzari S. Coop. de Iniciativa Social.

Garofalo, C. y Bogaerts, S. (2019). Attachment and Personality Disorders Among Child Molesters: The Role of Trust. Sexual Abuse, 31(1), 97-124.

Garrido, F., León, J., López, V. y Ojeda, F. (2020). Asociación de las conductas de riesgo en adolescentes. Estrategias para su prevención. Index de Enfermería, 28(3), 110-114. 
Garrido, L. (2006). Apego, Emocion y Regulacion Emocional. Implicaciones para la Salud. Revista Latinoamericana de Psicologia, 38(3), 493-507.

González, R., Ysern, L., Martorell, C., Matéu, C. y Barreto, P. (2010). Relaciones entre psicopatolodía y apego en la adolescencia. Revista Iberoamericana de Diagnostico y Evaluacion, 1(29), 9-26.

Guzmán, M., Carrasco, N., Figueroa, P., Trabucco, C. y Vilca, D. (2016). Estilos de Apego y Dificultades de Regulación Emocional. PSYKHE, 25(1), 1-13.

Hernandez, R. (2014). Metodologia de la Investigacion. Mexico: Mcgraw-HILL / Interamericana Editores, S.A. DE C.V.

Horno, P. (2014). Apego y Adolecencia: Narrándose en el espejo de los otros. Adolescere, 2(3), 19-28.

ICBF. (30 de 06 de 2018). Instituto Colombiano de Bienestar Familiar. Obtenido de https://www.icbf.gov.co/bienestar/observatorio-bienestar-ninez/tablero-srpa

Instituto Nacional de Estadistica (20 de 09 de 2019). INE. Obtenido de https://www.ine.es/dyngs/INEbase/es/operacion.htm?c=Estadistica_C\&ci$\mathrm{d}=1254736176795 \&$ menu $=$ ultiDatos\&idp $=1254735573206$

Jiménez Ornelas, R. (2005). La delincuencia juvenil: fenómeno de la sociedad actual. Papeles de población, 11(43), 215-261.

Lecannelier, F., Ascanio, L., Flores, F. y Hoffmann, M. (2011). Apego \& psicopatologia: Una Revisión Actualizada Sobre los Modelos Etiológicos Parentales del Apego Desorganizado. Terapia Psicológica, 29(1), 107-1016.

Levy K., Johnson B., Clouthier T., Scala J. y Temes C. (2015). An Attachment Theoretical Framework for Personality Disorders. Canadian Psychology, 56(2), $197-$ 207. doi:10.1037/cap0000025.

López, P. (2004). Poblacion Muestra y Muestreo. Punto Cero, 9(08), 69-74.

Mikulincer, M., Shaver, P. y Pereg, D. (2003). Attachment Theory and Affect Regulation: The Dynamics, Development, and Cognitive Consequences of Attachment-Related Strategies. Motivation and Emotion, 27, 77-102. https://doi. org/10.1023/A:1024515519160.

Miron, L., Luengo, A., Sobral, J. y Otero, J. (1988). Un analisis de la relacion entre ambiente familiar y delincuencia juvenil. Revista de Psicología Social, 3, 165-180.

Molina Sierra, G. (2018). Causas de reincidencia en los delitos de los menores en el SRPA, en la ciudad de Cartagena entre los años 2012 y 2015. Revista Jurídica Mario Alario D'Filippo, 10(19), 126-155. 
Calidad del apego percibido hacia la figura paterna, materna y pares en adolescentes infractores

Moneta, M. (2014). Apego y pérdida: redescubriendo a John Bowlby. Revista chilena de pediatría, 85(3), 265-268.

Mousalli, G. (2015). ResearchGate. Recuperado el 07 de 04 de 2020, de ResearchGate: https://www.researchgate.net/publication/303895876_Metodos_y_Disenos_de_Investigacion_Cuantitativa

Naupas, H., Valdivia, M., Palacios , J. y Romero, H. (2018). Metodología de la investigación. Bogota: Ediciones de la U.

Neves, S., Faraco, M. y Vieira, M. (2013). Attachment and Parental Practices as Predictors of Behavioral Disorders in Boys. Paidéia (Ribeirão Preto), 23(56), 369-377.

Nieri, L. (2017). Relación entre sensibilidad paterna y los estilos de apego, la personalidad y la capacidad empática, segun variables sociodemográficas. Psicologia desde el Caribe, 34(1), 1-15.

Niño, L. y Rivero, L. (2019). Legítima defensa, "la aplicación de criterios claros básico que se hacen necesarios para la configuración e interpretación de la legitima defensa, código penal colombiano, ley 599 de 2000, articulo 32-inciso 6" en el municipio de Arauca.

Oficina de Estadísticas de Justicia. (2019). Oficce of Juvenil Justice and Delinquency Prevention.

Paolicchi, G., Pennella, M., Colombres, R., Nuñez, M., Olivera, C., Abreu, L. y Sorgen, E. (2013). La construcción de los lazos entre generaciones: transmisión, apego y juego. Anuario de investigaciones, 20(2), 257-267.

Paramo, M. (2011). Factores de riesgo y factores de proteccion en la adolescencia: analisis de contenido a traves de grupos de discusion. Terapia psicologica, 29(1), 85-95. http://dx.doi.org/10.4067/S0718-48082011000100009.Perez, B. y Arrazola, T. (2013). Vínculo afectivo en la relación parento-filial como factor de calidad de vida. Revista Tendencias \& Retos, 18(1), 17-32.

Prado, M., Pineda, S., Carrillo, S. y Castro, J. (2006). Análisis Psicométrico del Inventario de Apego con Padres y Pares en una Muestra de Adolecentes Colombianos. Revista Interamericana de Psicología, 40(3), 289-302.

Ramos, V., Canta, G., de Castro, F. y Leal, I. (2016). The Relation Between Attachment, Personality, Internalizing, and Externalizing Dimensions in Adolescents With Borderline Personality Disorder. Bulletin of the Menninger Clinic, 80(3), 213-233.

Rendon, E. y Rodriguez, R. (2016). La importancia del vínculo en la infancia: entre el psicolanálisis y el neurobiología. Ciencias de la Salud, 14(2), 261-280. 
[22] Sergio Andrés Acosta Tobón, Samuel Colorado Muñoz, Sara Tahia Eusse Gallego, Jorge Mauricio Cuartas Arias

Sandoval , L. y Martínez, D. (2008). Una revision al estudio de la delincuencia y criminalidad. Revista Facultad de Ciencias Economicas: Investigacion y Reflexion, 16(1), 105-117.

Sarmiento, A., Puhl, S., Oteyza, G., Bermúdez, F. y Siderakis, M. (2009). Las conductas transgresoras de los adolescentes en conflicto con la ley penal; en su relacion con las perturbaciones en el vinculo primario. Anuario de investigaciones, $16,51-57$.

Sharp, C. y Fonagy, P. (2015). Practitioner Review: Borderline personality disorder in adolescence-Recent conceptualization, intervention, and implications for clinical practice. Journal of Child Psychology and Psychiatry, 56(12), 1266-1288. doi:10.1111/jcpp.12449.

Steele, M., Bate, J., Nikitiades, A. y Buhl-Nielsen, B. (2015). Attachment in Adolescence and Borderline Personality Disorder. Journal of Infant, Child, and Adolescent Psychotherapy, 14 (1), 16-32. DOI: 10.1080 / 15289168.2015.1004882

Unicef (2016). Sonadolescentes.org.uy. Obtenido de https://sonadolescentes.org.uy/ files/Adolescentes-y-sistemas-penales.pdf

Urrego, Y., Restrepo, J., Pinzon, S., Acosta , J., Diaz , M. y Bonilla , C. (2014). Vínculo Afectivo en Pares y cognición social en la infancia intermedia. International Journal Psychological Research, 7(2), 51-63.

Vega, L. (2010). Importancia de fomentar el vinculo de apego en la infancia. Revista Mexicana de Pediatría, 77(3), 103-104. 